N.P. Khuchua, N.D. Dolidze, N.G. Gapishvili, R.G. Gulyaev, Z.V. Jibuti, R.G. Melkadze and M.G. Tigishvili

\title{
Technology of semiconductor materials sensitive to different regions of the electromagnetic radiation spectrum
}

\begin{abstract}
Ion implantation (II) of impurities in a semiconductor followed by annealing belongs to the modern techniques that make it possible to modify the fundamental properties of a material, including optical ones, due to the formation of a great number of defects. It is an important factor for the creation of electromagnetic radiation (EMR) detectors. In the present work, in order to develop EMR detectors $\mathrm{p}-\mathrm{n}$ type semiconductor structures have been fabricated and studied. The p-layer was formed by implanting boron into commercial single-crystal n-type silicon at different doses $\left(1 \times 10^{13}-7.8 \times 10^{14} \mathrm{~cm}^{-2}\right)$ with an acceleration energy of $50 \mathrm{keV}$ and subsequent annealing at 800,900 and $1000{ }^{\circ} \mathrm{C}$ for $20 \mathrm{~min}$. Diode structures have been fabricated, current-voltage characteristics have been measured and the material sensitivity to EMR of different wavelengths has been studied. It has been shown that the ion-implanted material exhibits a well-marked photosensitivity in one of the most interesting IR $(1.4-2.2 \mu \mathrm{m})$ and UV $(0.2-0.4 \mu \mathrm{m})$ regions. Sensitivity to X-ray exposure has also been revealed. For interpretation of the results, the idea of the appearance of defects as clusters (nanoformations) in the silicon matrix is used. The novelty of the work is both in the experimental results and in the approach "from photosensitivity spectra to defect formation" since most other researchers concentrate their attention on the identification of defects appearing during impurity implantation in Si rather than on the optical properties of the material determined by these defects.
\end{abstract}

Keywords: boron ion-implanted silicon; clusters; infrared and ultraviolet regions; nanoformations; photosensitivity; X-radiation

Nanotechnology Perceptions 10 (2014) 91-99 